\title{
Effect of a Face Sheet Tool on Medical Team Provider Identification and Family Satisfaction
}

\author{
Ndidi I. Unaka, MD*, Christine M. White, MD, MAT, Heidi J. Sucharew, PhD, Connie Yau, BA, Stephanie L. Clark, MD, MPH, \\ Patrick W. Brady, MD, MSc
}

Division of Hospital Medicine, Cincinnati Children's Hospital Medical Center, Cincinnati, Ohio.

Resident duty hour restrictions may expose families to more trainees during hospitalization and hinder recognition of medical team members. This may negatively impact family satisfaction. Our study sought to determine the effects of a face sheet tool on families' identification and satisfaction rating of the medical team. One of 2 general pediatric units at a large academic center was assigned to intervention; the other served as the concurrent control. Families on the intervention unit were given a face sheet tool with medical team members' photos and role descriptions. Upon discharge, caregivers matched names, photos, and roles to providers they encountered, answered a 10-question satis- faction survey, and answered an overall hospital experience satisfaction question. Caregivers encountered a median of 8 (range, 3-14) medical team members. Caregivers in the intervention group were more likely to correctly identify providers by name (median correct, $25 \%$ vs $11 \%$ for controls; $P<0.01$ ) and provider roles (median correct, $50 \%$ vs $25 \%$; $P<0.01$ ). No significant difference was noted between groups for overall satisfaction. A face sheet tool helped caregivers identify their child's care providers' names and roles, although identification remained poor. Journal of Hospital Medicine 2014;9:186-188. (C) 2013 Society of Hospital Medicine.
Acute illness requiring hospitalization can be overwhelming for children and their families who are coping with illness and the synthesis of information from a variety of healthcare providers. ${ }^{1}$ Patient and family centeredness is endorsed by the Institute of Medicine and the American Academy of Pediatrics ${ }^{2,3}$ as central to quality healthcare. In academic institutions, the presence of medical students and residents adds to the number of providers families encounter. In July 2011, the Accreditation Council for Graduate Medical Education implemented new duty hour restrictions, limiting first year residents to a maximum of 16 hour shifts. ${ }^{4}$ Consequently, caregivers and patients may be in contact with more healthcare providers; this fractured care may confuse patients and caregivers, and increase dissatisfaction with care. ${ }^{5}$

The primary objective of our study was to determine the effect of a face sheet tool on the percentage of medical team members correctly identified by caregivers. The secondary objective was to determine the effect of a face sheet tool on the evaluation and satisfaction rating of the medical team by caregivers. We hypothesized that caregivers who receive the face sheet tool will correctly identify a greater percentage

*Address for correspondence and reprint requests: Ndidi I. Unaka, MD, Cincinnati Children's Hospital Medical Center, 3333 Burnet Ave. ML 5018, Cincinnati, OH 45229; Telephone: 513-636-8354; Fax: 513-6367905; E-mail: ndidi.unaka@cchmc.org

Additional Supporting Information may be found in the online version of this article.

Received: August 21, 2013; Revised: October 11, 2013; Accepted: October 21, 2013

2013 Society of Hospital Medicine DOI 10.1002/jhm.2114

Published online in Wiley Online Library (Wileyonlinelibrary.com). of team members by name and role and have higher overall satisfaction with their hospital stay.

\section{METHODS}

We performed a prospective controlled study on 2 general pediatric units at Cincinnati Children's Hospital Medical Center (CCHMC). Patients on the intervention unit received the face sheet tool, whereas the concurrent control unit maintained usual procedures. Both units have 24 beds and care for general pediatric patients primarily covered by 4 resident teams and the hospital medicine faculty. Two paired resident teams composed of 2 senior residents, 3 to 4 interns, and 4 medical students primarily admit to each general pediatric unit. Team members rotate through day and night shifts. All employees and rotating students are required to wear the hospital issued identification badge that includes their names, photos, credentials, and role. The study was conducted from November 1, 2011 to November 30, 2011.

Included patients were admitted to the study units by the usual protocol at our hospital, in which nurse patient-flow coordinators determine bed assignments. We excluded families whose children had an inpatient hospital stay of $<12$ hours and families who did not speak English. All patient families scheduled to be discharged later in the day on weekday mornings from the 2 study units were approached for study participation. Families were not compensated for their participation.

A face sheet tool, which is a sheet of paper with pictures and names of the intervention team attendings, senior residents, interns, and medical students as well as a description of team member roles, was distributed to patients and their caregivers. The face 
sheet tools were created using Microsoft Publisher (Microsoft Corp., Redmond, WA). Neither families nor providers were blinded to the intervention, and the residents assumed responsibility for introducing the face sheet tool to families.

For our primary outcome measure, the research coordinator asked participating caregivers to match provider photographs with names and roles by placing laminated pictures backed with Velcro tape in the appropriate position on a laminated poster sheet. Initially, we collected overall accuracy of identification by name and role. In the second week, we began collecting specific data on the attending physician.

The satisfaction survey consisted of the American Board of Internal Medicine (ABIM) patient satisfaction questionnaire, composed of 10,5-point Likert scale questions, ${ }^{6,7}$ and an overall rating of hospital question, "On a scale from 1 to 10 , with 1 being the worst possible hospital and 10 being the best possible hospital, what number would you rate this hospital?" from the Hospital Consumer Assessment of Health Plans Survey. ${ }^{8}$ Questions were asked aloud and families responded to the questions orally. A written list was also provided to families. We collected data on length of stay (LOS) at the time of outcome assessment as well as previous hospitalizations.

\section{Data Analysis}

Differences between the intervention and control groups for relationship of survey respondent to child, prior hospitalization, and LOS were evaluated using the Fisher exact, $\chi^{2}$, and 2-sample $t$ test, respectively. Hospital LOS was log-transformed prior to analysis. The effect of the face sheet tool was evaluated by analyzing the differences between the intervention and control groups in the proportion of correctly identified names and roles using the Wilcoxon rank sum test and using the Fisher exact test for attending identification. Skewed Likert scale satisfaction ratings and overall hospital ratings were dichotomized at the highest score possible and analyzed using the $\chi^{2}$ test. An analysis adjusting for prior hospitalization and LOS was done using generalized linear models, with a Poisson link for the number of correctly identified names/roles and an offset for the number of names/roles given.

Our research was reviewed by the CCHMC institutional review board and deemed exempt.

\section{RESULTS}

A total of 96 families were approached for enrollment (50 in the intervention and 46 in the control). Of these, 86 families agreed to participate. Three families in the intervention group did not receive the face sheet tool and were excluded from analysis, leaving an analytic cohort of 83 (41 in intervention and 42 in control). Attending recognition by role was collected from 54 families (28 in intervention group and 26 in control group) and by name from 34 families (15 in
TABLE 1. Family Characteristics by Group

\begin{tabular}{lccc}
\hline & Intervention, $\mathrm{n}=41$ & Control, $\mathrm{n}=42$ & $P$ Value $^{*}$ \\
\hline Relationship to patient & & & 0.67 \\
Mother & $33(80 \%)$ & $35(83 \%)$ & \\
Father & $5(12 \%)$ & $6(14 \%)$ & \\
Grandmother/legal guardian & $3(7 \%)$ & $1(2 \%)$ & \\
Prior hospitalization, yes & $12(29 \%)$ & $24(57 \%)$ & 0.01 \\
Length of stay (days) & $1.07(0.86-1.34)$ & $1.32(1.05-1.67)$ & 0.20 \\
\hline
\end{tabular}

NOTE: Data are expressed as $n(\%)$ or geometric mean ( $95 \%$ confidence interval).

${ }^{*} P$ values for the difference between groups are from $\chi^{2}$ test or Fisher exact test for categorical variables and 2-sample $t$ test for log length of stay.

intervention group and 19 in control group). Table 1 displays characteristics of each group. Among the 83 study participants, LOS at time of outcome assessment ranged from 0.4 to 12.0 days, and the number of medical team members that cared for these patients ranged from 3 to 14 .

Families in the intervention group had a higher percentage of correctly identified members of the medical team by name and role as compared to the control group (Table 2). These findings remained significant after adjusting for LOS and prior hospitalization. In addition, in a subset of families with attending data available, more families accurately identified attending name and attending role in the intervention as compared to control group.

No significant differences were noted between the groups when comparing all individual ABIM survey question scores or the overall hospital satisfaction rating (Table 2). Scores in both intervention and control groups were high in all categories.

\section{DISCUSSION}

Caregivers given the face sheet tool were better able to identify medical team members by name and role than caregivers in the control group. Previous studies have shown similar results. ${ }^{9,10}$ Families encountered a large number of providers (median of 8 ) during stays that were on average quite brief (median LOS of 23.6 hours). Despite the significant increase in caregivers' ability to identify providers, the effect was modest.

Our findings add to prior work on face sheet tools in pediatrics and internal medicine. ${ }^{9-11}$ Our study occurred after the residency duty hour restrictions. We described the high number of providers that families encounter in this context. It is the first study to our knowledge to quantify the number of providers that families encounter after these changes and to report on how well families can identify these clinicians by name and role. Unlike other studies, satisfaction scores were not improved. ${ }^{9}$ Potential reasons for this include: (1) caregiver knowledge of 2 to 4 key members of the team and not the whole team may be the primary driver of satisfaction, (2) caregiver activation or empowerment may be a more responsive measure than overall satisfaction, and (3) our satisfaction 


\begin{tabular}{|c|c|c|c|}
\hline & Intervention & Control & $P$ Value ${ }^{\star}$ \\
\hline Medical team, proportion correctly identified: & $N=41$ & $N=41$ & \\
\hline Medical team names & $25 \%(14,58)$ & $11 \%(0,25)$ & $<0.01^{\dagger}$ \\
\hline Medical team roles & $50 \%(37,67)$ & $25 \%(12,44)$ & $<0.01^{\dagger}$ \\
\hline \multicolumn{4}{|l|}{ Attending, correctly identified: } \\
\hline \multirow[t]{2}{*}{ Attending's name } & $N=15$ & $N=19$ & \\
\hline & $14(93 \%)$ & $10(53 \%)$ & $0.02^{\ddagger}$ \\
\hline \multirow[t]{2}{*}{ Attending's role } & $N=28$ & $N=26$ & \\
\hline & $26(93 \%)$ & $16(62 \%)$ & $0.01 \ddagger$ \\
\hline Patient satisfaction, best possible score for: & $N=41$ & $N=42$ & \\
\hline Q1: Telling you everything, being truthful & $21(51 \%)$ & $21(50 \%)$ & 0.91 \\
\hline Q2: Greeting you warmly, being friendly & $26(63 \%)$ & $25(60 \%)$ & 0.72 \\
\hline Q3: Treating you like you're on the same level & $29(71 \%)$ & $25(60 \%)$ & 0.28 \\
\hline Q4: Letting you tell your story, listening & $27(66 \%)$ & $23(55 \%)$ & 0.30 \\
\hline Q5: Showing interest in you as a person & $26(63 \%)$ & $23(55 \%)$ & 0.42 \\
\hline Q6: Warning your child during the physical exam & $21(51 \%)$ & $21(50 \%)$ & 0.91 \\
\hline Q7: Discussing options, asking your opinion & $20(49 \%)$ & $17(40 \%)$ & 0.45 \\
\hline Q8: Encouraging questions, answering clearly & $23(56 \%)$ & $19(45 \%)$ & 0.32 \\
\hline Q9: Explaining what you need to know & $22(54 \%)$ & $18(43 \%)$ & 0.32 \\
\hline Q10: Using words you can understand & $26(63 \%)$ & $18(43 \%)$ & 0.06 \\
\hline Overall hospital rating & $27(66 \%)$ & $26(62 \%)$ & 0.71 \\
\hline
\end{tabular}

NOTE: Data are expressed as median (25th, 75 th percentile) or $n(\%)$.

${ }^{*} P$ values from $\chi^{2}$ test unless noted otherwise.

${ }^{\dagger} P$ value from Wilcoxon rank sum test.

${ }^{\ddagger} P$ value from Fisher exact test.

measures may have ceiling effects and/or be elevated in both groups by social desirability bias.

Our study highlights the need for further investigation of quality outcomes associated with residency work hour changes. ${ }^{12-14}$ Specifically, exposure to large numbers of providers may hinder families from accurately identifying those entrusted with the care of their loved one. Of note, our research coordinator needed to present as many as 14 provider pictures to 1 family with a hospital stay of $<24$ hours. Large numbers of providers may create challenges in building rapport, ensuring effective communication and developing trust with families. We chose to evaluate identification of each team member by caregivers; our findings are suggestive of the need for alternative strategies. A more valuable intervention might target identification of key team members (eg, attending, primary intern, primary senior resident). A policy statement regarding transitions of care recommended the establishment of mechanisms to ensure patients and their families know who is responsible for their care. ${ }^{15}$ Efforts toward achieving this goal are essential.

This study has several limitations. The study was completed at a single institution, and thus generalizability may be limited. Although the intervention and control units have similar characteristics, randomization did not occur at the patient level. The control group had significantly more patients who had greater than 1 admission compared to the intervention group. Patients enrolled in the study were from a weekday convenience sample; therefore, potential differences in results based on weekend admissions were unable to be assessed. The exclusion of non-English-speaking families could limit generalizability to this population. Social desirability bias may have elevated the scores in both groups. Providers tasked with the responsibility of introducing the face sheet tool to families did so in a nonstandardized way and may have interacted differently with families compared to the control team. Finally, our project's aim was focused on the effect of a face sheet tool on the identification and satisfaction rating of the medical team by caregivers. Truly family-centered care would include efforts to improve families' knowledge of and satisfaction with all members of the healthcare team.

A photo-based face sheet tool helped caregivers better identify their child's care providers by name and role in the hospital. Satisfaction scores were similar in both groups.

\section{Acknowledgements}

The authors thank the Pediatric Research in Inpatient Settings network, and specifically Drs. Karen Wilson and Samir Shah, for their assistance during a workshop at the Pediatric Hospital Medicine 2012 meeting in July 2012, during which a first draft of this manuscript was produced.

Disclosure: Nothing to report.

\section{References}

1. Diaz-Caneja A, Gledhill J, Weaver T, Nadel S, Garralda E. A child's admission to hospital: a qualitative study examining the experiences of parents. Intensive Care Med. 2005;31(9):1248-1254.

2. Committee on Quality of Health Care in America. Institute of Medicine. Crossing the Quality Chasm: A New Health System for the 21st Century. Washington, DC: National Academy Press; 2001.

3. Committee on Hospital Care and Institute for Patient- and FamilyCentered Care. Patient- and family-centered care and the pediatrician's role. Pediatrics. 2012;129(2):394-404.

4. Nasca TJ, Day SH, Amis ES Jr. The new recommendations on duty hours from the ACGME Task Force. N Engl J Med. 2010;363(2):e3.

5. Latta LC, Dick R, Parry C, Tamura GS. Parental responses to involvement in rounds on a pediatric inpatient unit at a teaching hospital: a qualitative study. Acad Med. 2008;83(3):292-297.

6. PSQ Project Co-Investigators. Final Report on the Patient Satisfaction Questionnaire Project. Philadelphia, PA: American Board of Internal Medicine; 1989.

7. Brinkman WB, Geraghty SR, Lanphear BP, et al. Effect of multisource feedback on resident communication skills and professionalism: a randomized controlled trial. Arch Pediatr Adolesc Med. 2007;161(1): 44-49.

8. Giordano LA, Elliott MN, Goldstein E, Lehrman WG, Spencer PA. Development, implementation, and public reporting of the HCAHPS survey. Med Care Res Rev. 2010;67(1):27-37.

9. Dudas RA, Lemerman H, Barone M, Serwint JR. PHACES (Photographs of Academic Clinicians and Their Educational Status): a tool to improve delivery of family-centered care. Acad Pediatr. 2010;10(2): $138-145$.

10. Arora VM, Schaninger C, D'Arcy M, et al. Improving inpatients' identification of their doctors: use of FACE cards. Jt Comm J Qual Patient Saf. 2009;35(12):613-619.

11. Amer A, Fischer H. "Don't call me 'mom'": how parents want to be greeted by their pediatrician. Clin Pediatr. 2009;48(7):720-722.

12. Auger KA, Landrigan CP, Gonzalez Del Rey JA, Sieplinga KR, Sucharew HJ, Simmons JM. Better rested, but more stressed? Evidence of the effects of resident work hour restrictions. Acad Pediatr. 2012; 12(4):335-343.

13. Gordon MB, Sectish TC, Elliott MN, et al. Pediatric residents' perspectives on reducing work hours and lengthening residency: a national survey. Pediatrics. 2012;130(1):99-107.

14. Oshimura J, Sperring J, Bauer BD, Rauch DA. Inpatient staffing within pediatric residency programs: work hour restrictions and the evolving role of the pediatric hospitalist. J Hosp Med. 2012;7(4):299303.

15. Snow V, Beck D, Budnitz T, et al. Transitions of Care Consensus policy statement: American College of Physicians, Society of General Internal Medicine, Society of Hospital Medicine, American Geriatrics Society, American College of Emergency Physicians, and Society for Academic Emergency Medicine. J Hosp Med. 2009;4(6):364-370. 